\title{
The usage of biological DMARDs and clinical remission of rheumatoid arthritis in China: a real-world large scale study
}

\author{
Yuan $\mathrm{An}^{1} \cdot$ Tian Liu ${ }^{1} \cdot$ Dongyi He${ }^{2} \cdot$ Lijun $\mathrm{Wu}^{3} \cdot{\mathrm{Juan} \mathrm{Li}^{4} \cdot \mathrm{Yi} \mathrm{Liu}}^{5} \cdot \mathrm{Liqi}^{4}{ }^{6}$. \\ Bin $\mathrm{Zhou}^{7} \cdot$ Changsong $\mathrm{Lin}^{8} \cdot \mathrm{Lan} \mathrm{He}^{9} \cdot$ Xiangyuan $\mathrm{Liu}^{10} \cdot$ Xiaofeng $\mathrm{Li}^{11}$. \\ Niansheng Yang ${ }^{12}$. Zhuoli Zhang ${ }^{13} \cdot$ Hui Song ${ }^{14}$ - Wei Wei ${ }^{15} \cdot$ Jing Liu $^{16} \cdot$ Yu Bi $^{16}$. \\ Zhanguo Li ${ }^{1}$
}

Received: 23 March 2016/Revised: 8 August 2016 /Accepted: 18 September 2016/Published online: 5 October 2016

(C) The Author(s) 2016. This article is published with open access at Springerlink.com

\begin{abstract}
The aims of this study are to characterize the biological disease-modifying antirheumatic drug (bDMARD) usage patterns in real-life and examine the remission rate of rheumatoid arthritis (RA) patients receiving bDMARDs in routine clinical practice in China. Consenting RA patients ( $\geq 18$ years) from 15 teaching hospitals and receiving marketed bDMARDs were included. In total, 802 patients ( $81.3 \%$ women, $49.0 \pm 13.9$ years) were included; $89.5 \%$ were receiving a combination of bDMARDs and conventional synthetic DMARDs (csDMARDS), whereas $10.5 \%$ were receiving bDMARD monotherapy. Etanercept (including Enbrel ${ }^{\circledR}$ and local brand Yi Sai Pu® and Qiangke $\left.®\right)$, tocilizumab, adalimumab, and infliximab were used by $66.6 \%, 17.0 \%, 7.5 \%$, and $6.6 \%$ patients, respectively. Etanercept was used at a mean
\end{abstract}

Yuan An and Tian Liu contributed equally to this work.

Zhanguo Li

zgli99@aliyun.com

1 Department of Rheumatology and Immunology, Peking University People's Hospital, 11 South Xi Zhi Men Street, Xicheng District, Beijing 100044, People's Republic of China

2 Department of Rheumatology, Shanghai Guanghua Hospital, Shanghai, China

3 Department of Rheumatology, People's Hospital of Xinjiang Uygur Autonomous Region, Xingiiang, China

4 Department of Rheumatology, Nanfang Hospital of Southern Medical University, Guangzhou, China

5 Department of Rheumatology and Immunology, West China Hospital, West China School of Medicine, Sichuan University, Chengdu, China

6 Department of Rheumatology, China-Japan Union Hospital of Jilin University, Changchun, China

7 Department of Rheumatology, Sichuan Provincial People's Hospital, Sichuan, China weekly dose of $38.2 \pm 15.6 \mathrm{mg}$ for $25.5 \pm 47.0$ weeks and tocilizumab at $94.5 \pm 21.9 \mathrm{mg}$ for $4.7 \pm 7.5$ weeks. Overall rate of remission was $12.6 \%, 5.4 \%$, and $3.5 \%$ based on DAS28, CDAI, and SDAI scores, respectively. Compared with patients receiving bDMARDs for $<3$ months, those receiving bDMARDs for $\geq 3$ months exhibited significantly lower DAS28 scores $(p<0.0001)$, and a significantly higher proportion of patients who received bDMARDs for $\geq 12$ months achieved the treatment goal (remission or low disease activity, $62.5 \%$ vs. $18.3 \%$, $p<0.0001$ ). Patients receiving combination therapy with csDMARDs exhibited lower DAS28 scores than patients receiving bDMARD monotherapy ( 4.3 vs. $4.8, p=0.011)$. This large-scale real-world study showed that bDMARD usage
8 Department of Rheumatology, The First Affiliated Hospital of Guangzhou Traditional Chinese Medicine University, Guangzhou, China

9 Department of Rheumatology, The First Affiliated Hospital of Xi'An Jiaotong University, Xi'an, China

10 Department of Rheumatology, Peking University Third Hospital, Beijing, China

11 Department of Rheumatology, The Second Hospital of Shanxi Medical University, Taiyuan, China

12 Department of Rheumatology, The First Affiliated Hospital of Sun Yat-sen University, Guangzhou, China

13 Department of Rheumatology, Peking University First Hospital, Beijing, China

14 Department of Rheumatology, Beijing Jishuitan Hospital, Beijing, China

15 Department of Rheumatology, Tianjin Medical University General Hospital, Tianjin, China

16 Shanghai Roche Pharmaceuticals Ltd, Shanghai, China 
patterns in routine clinical practice in China were in accordance with international guidelines for RA management despite the short treatment duration. Longer duration of bDMARD usage and combination therapy showed a favored outcome of RA.

Keywords Biological $\cdot$ China $\cdot$ DMARD $\cdot$ Duration . Rheumatoid arthritis · Therapy

\section{Introduction}

Rheumatoid arthritis (RA) is a chronic systemic autoimmune disease associated with progressive joint damage and disability. The prevalence of RA is $0.24 \%$ globally and between 0.3 and $1 \%$ in developed countries [1, 2]. In Mainland China, the prevalence of RA has been reported to be $0.28 \%[3,4]$. As shown by recent studies, RA is becoming one of the most common disabling and costly disease in China [5-7]. Chou et al. reported a significantly higher RA prevalence in urban areas than in rural areas of Taiwan [8]. Socioeconomic and genetic factors might contribute to the lower prevalence in China compared with Western countries [9].

Disease-modifying antirheumatic drugs (DMARDs) are the standard treatment for patients with RA [10]. In addition to conventional synthetic DMARDs (csDMARDs), such as methotrexate, biological DMARDs (bDMARDs), including inhibitors targeting tumor necrosis factor (TNF)- $\alpha$, T cells, B cells, and interleukin- 6 , have been increasingly used in recent years $[10$, 11]. According to the 2013 European League Against Rheumatism (EULAR) and the 2012 American College of Rheumatology (ACR) recommendations, csDMARDs such as methotrexate should be chosen as the first-line treatment $[10$, 11]. However, the recommendations suggest bDMARDs for patients who fail to achieve low disease activity (LDA) or remission after receiving csDMARDs $[10,11]$. Although a combination therapy of csDMARDs and bDMARDs is recommended and commonly practiced, real-life registry data show that approximately $30 \%$ of patients receive bDMARDs as monotherapy in Western countries [12]. In a double-blind randomized trial, Dougados et al. found that the efficacy of tocilizumab monotherapy was comparable to a combined therapy of tocilizumab plus methotrexate in patients who had insufficient responses to methotrexate [13]. Emery et al. systematically reviewed trials evaluating bDMARD monotherapy and found that only tocilizumab monotherapy appeared to show efficacy equivalent to combination therapy with methotrexate [12]. In a recent large observational study, Gabay et al. found that patients receiving tocilizumab with or without concomitant csDMARD showed comparable clinical response [14].

Until the end of patient enrollment of this study, a total of six bDMARDs have been approved for use in RA in China, including the interleukin-6 receptor inhibitor, tocilizumab, and five TNF inhibitors (infliximab, adalimumab, and etanercept, including
Enbrel ${ }^{\circledR}$ and local brand Yi Sai Pu ${ }^{\circledR}$ and Qiangke $\left.{ }^{\circledR}\right)$. In recent years, bDMARDs have been increasingly prescribed in routine clinical practice in China. However, the "real-world" data on bDMARD usage patterns in China might be different from those in developed countries owing to socioeconomic factors. The objective of this cross-sectional study was to characterize the usage patterns of bDMARDs in Chinese patients with RA and examine the association between disease activity and clinical remission, and the duration and pattern of bDMARD therapy, with the goal to optimize the management of RA in China.

\section{Materials and methods}

\section{Study design}

This multicenter observational cross-sectional study was conducted in 15 hospitals across different regions of China. The study was approved by the Institutional Review Board of Peking University and independent ethics committees responsible for each investigating site. The study was conducted in compliance with the Declaration of Helsinki and in accordance with Good Clinical Practice and relevant ethical guidelines. Written informed consent was obtained from each participant.

\section{Participants}

Patients aged $\geq 18$ years with confirmed RA according to the ACR 1987 criteria [15] and receiving bDMARDs at the time of interview were enrolled in the study. Patients were excluded if their physicians believed that the patients were not appropriate to participate in the study. Non-consenting patients were excluded.

\section{Data collection}

Questionnaires were administered through face-to-face interviews in each center by trained participating investigators with eligible patients. Data on age, sex, RA duration, total joint count, swollen joint count, and visual analogue scale (VAS) were collected on enrollment visit. The levels of C-reactive protein, hemoglobin, erythrocyte sedimentation rate, rheumatoid factor, and anti-cyclic citrullinated peptides measured within 2 weeks of the enrollment visit were obtained from medical records. Disease activity was evaluated according to the Disease Activity Score based on 28-joint count (DAS28) (remission: DAS2 $<2.6$; low disease activity: $2.6 \leq$ DAS $28<3.2$; moderate disease activity: $3.2 \leq$ DAS $28<5.1$; high disease activity: DAS28 $\geq 5.1[16,17])$, the Simplified Disease Activity Index (SDAI) (remission: SDAI $\leq 3.3$; low disease activity: $3.3<$ $\mathrm{SDAI} \leq 11$; moderate disease activity: $11<\mathrm{SDAI} \leq 26$; high disease activity: SADI $>26$ ), and the Clinical Disease Activity Index (CDAI) (remission: $\mathrm{CDAI} \leq 2.8$; low disease activity: $2.8<\mathrm{CDAI} \leq 10$; moderate disease activity: $10<\mathrm{CDAI} \leq 22$; 
high disease activity: CDAI $>22$ ).). Quality of life of patients was assessed using health assessment questionnaire disability index (HAQ-DI) on enrollment visit. Visual analog scales on enrollment visit (from 0 to $10 \mathrm{~cm}$ ) were used to assess fatigue and pain levels, with increasing values representing more severe fatigue and pain.

Data on usage patterns of bDMARDs, including information on monotherapy or combination therapy, the drug(s) used, frequency, treatment duration, and dosage of bDMARDs, were collected. The reasons for discontinuation or switching of bDMARDs and information about previous bDMARDs, including drug name and treatment duration, were recorded. All the AE information of using the common biologic agents was recorded and followed until return to stabilized.

\section{Sample size estimation}

The formula to calculate sample size in a prevalence survey was used $[18,19]$. Based on European and US registry data, approximately one third (33\%) of RA patients receive bDMARDs as monotherapy. Thus, with the assumption that the proportion of patients in China receiving bDMARD monotherapy is also $33 \%$ and a confidence level of $95 \%$ with maximum relative error of $10 \%$, the required sample size was estimated to be 800 .

\section{Statistical analysis}

The statistical analysis was performed using the statistical software SAS 9.2 (Cary, North Carolina, USA). Continuous data are presented as mean and standard deviation (SD), median, and range. Two-group comparisons were performed using Student $t$ test ( $p$ values were two-sided with a significance level of 0.05 ). Multiple-group comparisons were performed by analysis of variance with Bonferroni correction. Laboratory tests performed in different hospitals were standardized by statistical adjustment [20]. Categorical data, tabulated as frequencies and percentages, were analyzed using chi-square test. Missing values were excluded from the analyses.

\section{Results}

\section{Patient characteristics and bDMARD usage patterns}

A total of 808 patients were enrolled between December 2013 and August 2014. Six patients were excluded for violating the inclusion criteria (five patients were diagnosed with ankylosing spondylitis and one patient was $<18$ years old). Available data from 802 patients were analyzed. As shown in Table 1, patients (mean age of $49.0 \pm 13.9$ years) had a mean disease course of $3.2 \pm 5.8$ years. Abnormal C-reactive protein and erythrocyte sedimentation rate levels were exhibited by 60.8 $\%$ and $70.1 \%$ of patients, respectively. The majority of the patients were positive for rheumatoid factor $(77.6 \%)$ or anticyclic citrullinated peptides $(83.2 \%)$. Disease activity in the patients varied widely, as reflected by a broad range of DAS28, CDAI, and SDAI scores. The patients reported good quality of life and medium levels of fatigue and pain.

In the current study, etanercept was used by $66.6 \%$ (including Yi Sai Pu® $58.1 \%$, Enbrel® $6.1 \%$, and Qiangke ${ }^{\circledR}$ $2.4 \%$ ) patients. Tocilizumab, adalimumab, and infliximab were used by $17.0 \%, 7.5 \%$, and $6.6 \%$ of patients, respectively. The mean weekly doses and durations of bDMARDs are shown in Table 2. Only $10.5 \%$ of patients were receiving bDMARD monotherapy, amongst who, $75.0 \%, 10.7 \%, 9.5$ $\%$, and $4.8 \%$ were using etanercept, infliximab, tocilizumab, and adalimumab, respectively. The remaining patients $(89.5 \%)$ were receiving combination therapy of csDMARDs and bDMARDs. Most patients received one (49.3\%) or two (41.2\%) csDMARDs, whereas only $9.5 \%$ were on three csDMARDs. Among the patients on combination therapy, the proportion of patients using etanercept, infliximab, tocilizumab, or adalimumab was $65.7 \%, 8.4 \%, 18.1 \%$, and $7.8 \%$, respectively; these proportions were similar to those of patients on bDMARD monotherapy. The dose and duration of bDMARDs in combination therapy were comparable to those in bDMARD monotherapy (Table 3). The most commonly administered concomitant csDMARD was methotrexate, used by $65.9 \%$ of patients at a mean weekly dose of $9.8 \pm 2.8 \mathrm{mg}$ for $63.4 \pm 120.8$ weeks. Furthermore, $41.8 \%$ and $41.5 \%$ of patients were using concomitant hydroxychloroquine (2382.1 $\pm 674.1 \mathrm{mg} /$ week $)$ and leflunomide $(107.8 \pm 36.4 \mathrm{mg} /$ week $)$, respectively. In addition to csDMARDs, other types of drugs, including nonsteroidal anti-inflammatory drugs (NSAIDs), glucocorticoids, and topical drugs, were concomitantly used by $56.1 \%, 29.7 \%$, and $19.1 \%$ of patients, respectively (Table 3).

The three top reasons for discontinuing bDMARDs $(n=58)$ were clinical improvement $(31.0 \%)$, financial burden (24.1\%), and AEs (13.8\%). Among patients switching to different bDMARDs $(n=93)$, the main reasons for switching were unsatisfactory efficacy of the previous bDMARD $(58.1 \%)$, AEs (14.0\%), improvement of disease condition $(10.8 \%)$, and financial burden $(10.8 \%)$. Among the 802 patients, only $5(0.6 \%)$ reported at least one AE after initiation of this study, including one case of mild pruritus and another case of rash, which were suspected to be associated with etanercept. No bDMARDs-related serious AE was reported. Further analyses of disease activity revealed that the overall rate of remission was $12.6 \%, 5.4 \%$, and $3.5 \%$ based on DAS28, CDAI, and SDAI scores, respectively.

\section{Short duration of bDMARD therapy was associated with poor management of RA}

The duration of bDMARD therapy in the patients varied from 0.1 to 350.0 weeks (Table 2). Patients receiving bDMARDs 
Table 1 Patient characteristics $(N=802)$

\begin{tabular}{|c|c|c|c|}
\hline & Patients, $n(\%)$ & Mean (SD) & Median (range) \\
\hline Age (year) & 800 & $49.0(13.9)$ & $50.3(18.2-84.2)$ \\
\hline \multicolumn{4}{|l|}{ Gender, $n(\%)$} \\
\hline Men & $150(18.7)$ & & \\
\hline Women & $652(81.3)$ & & \\
\hline Body weight (kg) & 799 & $58.9(10.6)$ & $58.0(30.0-104.0)$ \\
\hline \multicolumn{4}{|l|}{ RA status } \\
\hline Disease course $^{\mathrm{a}}$ (years) & 357 & $3.2(5.8)$ & $0.6(0.0-40.3)$ \\
\hline $0.0-0.5, n(\%)$ & $176(49.3)$ & & \\
\hline $0.5-10, n(\%)$ & $139(38.9)$ & & \\
\hline More than $10, n(\%)$ & $42(11.8)$ & & \\
\hline $\mathrm{CRP}(\mathrm{mg} / \mathrm{mL})$ & 344 & $27.7(33.9)$ & $12.7(0.1-210.0)$ \\
\hline Abnormal $^{\mathrm{b}}, n(\%)$ & $209(60.8)$ & & \\
\hline ESR (mm/hour) & 394 & $42.4(31.1)$ & $35.5(1.0-140.0)$ \\
\hline Abnormal $^{\mathrm{b}}, n(\%)$ & $276(70.1)$ & & \\
\hline Hemoglobin (g/L) & 411 & $117.1(18.8)$ & $118.3(63.7-165.2)$ \\
\hline Anemia $^{\mathrm{c}}, n(\%)$ & $147(35.8)$ & & \\
\hline RF, $n$ (positive \%) & $184(77.6)$ & & \\
\hline ACCP, $n$ (positive \%) & $144(83.2)$ & & \\
\hline DAS28 & 412 & $4.4(1.5)$ & $4.4(0.5-7.7)$ \\
\hline CDAI & 801 & $20.2(15.3)$ & $16.0(0.0-76.0)$ \\
\hline SDAI & 343 & $27.2(18.1)$ & $23.9(0.13-83.5)$ \\
\hline TJC & 802 & $6.7(7.2)$ & $4.0(0.0-28.0)$ \\
\hline SJC & 802 & $4.8(6.1)$ & $2.0(0.0-28.0)$ \\
\hline \multicolumn{4}{|l|}{ Quality of life assessment } \\
\hline HAQ-DI & 801 & $11.9(13.4)$ & $7.0(0.0-60.0)$ \\
\hline VAS-fatigue & 801 & $3.7(2.5)$ & $3.5(0.0-10.0)$ \\
\hline VAS-pain & 801 & $4.2(2.4)$ & $4(0.0-10.0)$ \\
\hline
\end{tabular}

$S D$ standard deviation, $R A$ Rheumatoid arthritis, $C R P$ C-reactive protein $E S R$ Erythrocyte sedimentation rate, $R F$ Rheumatoid factor, $A C C P$ Anti-cyclic citrullinated peptide, $D A S 28$ Disease activity score based on 28 -joint count, $C D A I$ Clinical disease activity index, SDAI Simplified disease activity index, TJC Tender joint count, SJC Swollen joint count, $H A Q-D I$ Health assessment questionnaire disability index, VAS Visual analog scale

${ }^{\text {a }}$ Disease course was calculated according to the following equation: (the date when a patient signed the informed consent - the data when RA was diagnosed + 1)/365.25

${ }^{\mathrm{b}}$ The definitions of abnormal CRP and ESR follow participating hospitals' standardized criteria

${ }^{\mathrm{c}}$ Anemia was defined as hemoglobin $<120 \mathrm{~g} / \mathrm{L}$ for men and hemoglobin $<110 \mathrm{~g} / \mathrm{L}$ for women for 3.0-5.9 months or for $>12$ months had significantly lower DAS28 scores than those receiving bDMARDs $<3$ months ( $p=0.0002$ and $p<0.0001$, respectively, Table 4$)$. A significantly larger proportion of patients with $\geq 12$ months bDMARD therapy (DAS28 62.5\%, SDAI $63.6 \%$, CDAI
$62.9 \%$ ) achieved treatment target (LDA or remission) compared with patients with $<12$ months (DAS28 $21.0 \%$, SDAI $15.2 \%$, CDAI $25.8 \%$, all $p<0.05$ ). In contrast, the proportion of patients achieving treatment target was significantly lower in patients with $<3$ months bDMARDs (SDAI $12.2 \%$, CDAI
Table 2 Dose and treatment duration of bDMARD therapy

\begin{tabular}{|c|c|c|c|c|c|}
\hline \multirow[t]{2}{*}{ bDMARD } & \multirow[t]{2}{*}{ Patients, $n(\%)$} & \multicolumn{2}{|c|}{ Weekly dose (mg) } & \multicolumn{2}{|c|}{ Treatment duration (weeks) } \\
\hline & & Mean (SD) & Median (range) & Mean (SD) & Median (range) \\
\hline Etanercept & $534(66.6)$ & $38.2(15.6)$ & $50(2.1-100.0)$ & $25.5(47.0)$ & $6.0(0.1-350.0)$ \\
\hline Tocilizumab & $136(17.0)$ & $94.5(21.9)$ & $100(30.0-160.0)$ & $4.7(7.5)$ & $1.0(0.1-34.0)$ \\
\hline Adalimumab & $60(7.5)$ & $20.1(6.4)$ & $20.0(5.0-40.0)$ & $12.7(19.4)$ & $4.0(0.1-104.0)$ \\
\hline Infliximab & $53(6.6)$ & $33.1(23.6)$ & $25.0(3.8-100.0)$ & $34.5(39.1)$ & $24.0(0.1-186.0)$ \\
\hline
\end{tabular}


Table 3 bDMARD monotherapy and combination therapy $(N=802)$

\begin{tabular}{|c|c|c|c|c|}
\hline \multirow[t]{2}{*}{ Therapy type } & \multirow{2}{*}{$\begin{array}{l}\text { Patients } \\
n(\%)\end{array}$} & \multirow{2}{*}{$\begin{array}{l}\text { No. of patients with available } \\
\text { therapy duration }\end{array}$} & \multicolumn{2}{|c|}{ Duration (week) } \\
\hline & & & Mean (SD) & Median (range) \\
\hline bDMARD monotherapy ${ }^{\mathrm{b}}$ & $84(10.5)^{\mathrm{a}}$ & 84 & $27.4(54.6)$ & $1.5(0.1-240.0)$ \\
\hline Etanercept & $63(75.0)$ & 63 & $29.0(58.2)$ & $1.0(0.1-240.0)$ \\
\hline Infliximab & $9(10.7)$ & 9 & $42.0(60.8)$ & $24.0(0.1-186.0)$ \\
\hline Tocilizumab & $8(9.5)$ & 8 & $8.0(9.8)$ & $4.1(0.1-28)$ \\
\hline Adalimumab & $4(4.8)$ & 4 & $7.4(13.7)$ & $0.8(0.1-28.0)$ \\
\hline bDMARD + csDMARD combination therapy ${ }^{\mathrm{c}}$ & $718(89.5)^{\mathrm{a}}$ & 718 & $21.1(39.7)$ & $4.4(0.1-350.0)$ \\
\hline Etanercept & $472(65.7)$ & 472 & $25.0(45.4)$ & $6.0(0.1-350.0)$ \\
\hline Infliximab & $60(8.4)$ & 60 & $33.3(35.3)$ & $23.0(0.1-165.0)$ \\
\hline Tocilizumab & $130(18.1)$ & 130 & $4.5(7.3)$ & $1.0(0.1-34.0)$ \\
\hline Adalimumab & $56(7.8)$ & 56 & $13.1(19.8)$ & $4.0(0.1-104.0)$ \\
\hline Number of concomitant csDMARDs & $718(100)$ & & & \\
\hline One kind of csDMARDs & $354(49.3)$ & & NA & NA \\
\hline Two kinds of csDMARDs & $296(41.2)$ & & NA & NA \\
\hline Three kinds of csDMARDs & $68(9.5)$ & & NA & NA \\
\hline Type of concomitant csDMARDs ${ }^{\mathrm{d}}$ & $718(100)$ & & & \\
\hline Methotrexate & $473(65.9)$ & 468 & $63.4(120.8)$ & $16.0(0.1-999)$ \\
\hline Hydroxychloroquine & $300(41.8)$ & 298 & $37.3(58.5)$ & $10.0(0.1-364.0)$ \\
\hline Leflunomide & $299(41.6)$ & 296 & $59.1(82.6)$ & $28.0(0.1-520.0)$ \\
\hline Sulfasalazine & $46(6.4)$ & 45 & $61.3(148.6)$ & $12.0(0.1-750.0)$ \\
\hline Others & $34(4.7)$ & 32 & NA & NA \\
\hline Other concomitant drugs & $802(100)$ & & NA & NA \\
\hline Nonsteroidal anti-inflammatory drugs & $450(56.1)$ & & NA & NA \\
\hline Glucocorticoids $^{\mathrm{e}}$ & 238 (29.7) & & NA & NA \\
\hline Topical drugs & $153(19.1)$ & & NA & NA \\
\hline
\end{tabular}

${ }^{\text {a }}$ Percentage was calculated using the total number of patients $(N=802)$ as the denominator

${ }^{\mathrm{b}}$ bDMARD monotherapy represents bDMARD alone without combination with csDMARDs; the percentage of each bDMARD was calculated using the total number of patients on monotherapy $(n=84)$ as the denominator

${ }^{c}$ bDMARD + csDMARD combination therapy represents combination of bDMARDs and csDMARDs; the percentage of each bDMARD was calculated using the total number of patients on combination therapy $(n=718)$ as the denominator

${ }^{\mathrm{d}}$ The percentage of each type of csDMARD was calculated using the total number of patients on bDMARD $+\operatorname{csDMARD}$ combination therapy $(n=718)$ as the denominator

${ }^{\mathrm{e}}$ Among the 238 patients on concomitant glucocorticoids, 73.1 and $23.1 \%$ patients were receiving oral prednisone and methylprednisolone at a mean (SD) weekly dose (mg) of 57.8 (31.9) and 59.7 (151.6), respectively

$19.5 \%$ ) than in those with 3.0-5.9 months bDMARDs (SDAI $35.7 \%$, CDAI $40.7 \%$, all $p<0.0083$, Fig. 1$)$. The proportion of patients achieving treatment target was not significantly different between patients with 3-5.9 months bDMARDs and patients with 6.0-11.9 months (all $p>0.0083$, Fig. 1).

\section{The effects of treatment regimen on disease activity}

Patients receiving combination therapy of bDMARDs and csDMARDs showed significantly lower mean DAS28 scores than patients on bDMARD monotherapy (4.3 vs. 4.8, $p=0.0108$, Table 5). However, the percentage of patients achieving treatment target was not significantly different
Table 4 Association of bDMARD therapy duration and DAS28 scores

\begin{tabular}{llll}
\hline $\begin{array}{l}\text { Treatment duration } \\
\text { (months) }\end{array}$ & $\begin{array}{l}\text { Patients }^{\mathrm{a}} \\
(n=412)\end{array}$ & $\begin{array}{l}\text { Mean score } \\
(\mathrm{SD})\end{array}$ & $p$ value \\
\hline $0.0-2.9$ & 312 & $4.6(1.5)$ & \\
$3.0-5.9$ & 35 & $3.5(1.4)$ & $0.0002^{*}$ \\
$6.0-11.9$ & 25 & $3.9(1.4)$ & $0.0945^{*}$ \\
$>12$ & 40 & $3.2(1.4)$ & $<0.0001^{*}$ \\
\hline
\end{tabular}

*Compared with 0.0-2.9-month group, with analysis of variance followed by Bonferroni correction. The adjusted $\alpha=0.05 / 6=0.0083$; $* p<0.0083$

${ }^{\text {a }}$ Number of patients with available score data 


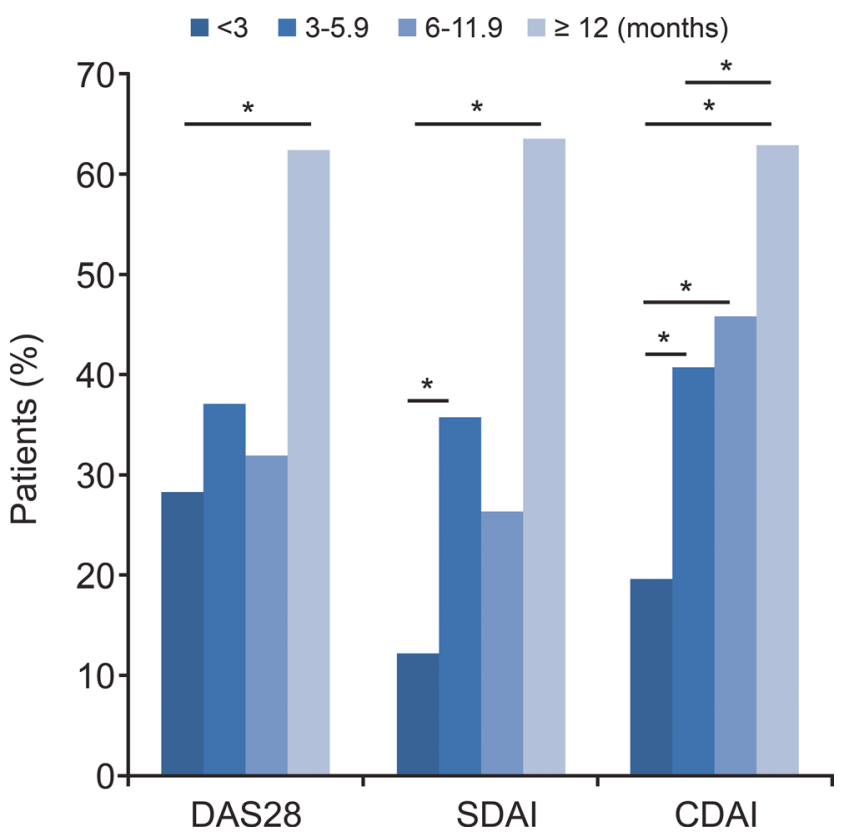

Fig. 1 The proportion of patients achieving treatment goal with various durations of bDMARDs. Comparison was performed by multiple comparisons with Bonferroni adjustments (the adjusted $\alpha=0.05$ / $6=0.0083 ; * p<0.0083$ )

between bDMARD monotherapy and combination therapy ( $21.4 \%$ vs. $25.6 \%, p=0.507$, Table 5). Further examination of patients with bDMARD monotherapy revealed that DAS28 score was significantly lower in patients receiving tocilizumab than in patients receiving TNF inhibitors $(3.6 \pm 2.1$ vs. 5.0 $\pm 1.7, p=0.0479$, Table 5). Consistently, significantly higher proportion of patients with tocilizumab monotherapy reached treatment target compared with patients receiving TNF inhibitor type of bDMARDs $(57.1 \%$ vs. $16.3 \%, p=0.0317$, Table 5). Notably, the DAS28 score in patients receiving TNF inhibitors as monotherapy was significantly higher than that in patients receiving combination therapy of TNF inhibitors and csDMARDs $(5.0 \pm 1.7$ vs. $4.3 \pm 1.4, p=0.0012$, Table 5), suggesting that TNF inhibitor monotherapy may not be as effective as combination therapy of TNF inhibitors plus csDMARDs. In contrast, DAS28 score was not significantly different in patients receiving tocilizumab monotherapy and patients receiving combination therapy of tocilizumab and csDMARDs (3.6 \pm 2.1 vs. $4.3 \pm 1.6, p=0.2672$, Table 5 ).

\section{Discussion}

This is the first large-scale multicenter study to characterize real-life bDMARD usage patterns and disease activity in routine practice in Chinese patients with RA receiving bDMARDs. The results show that combination therapy of bDMARDs and csDMARDs was substantially more common than bDMARD monotherapy in real-world clinical practice in China. Methotrexate was the predominant csDMARD administered to Chinese patients. The usage pattern of bDMARDs in China is in accordance with the guidelines for management of $\mathrm{RA}$, in which methotrexate is recommended as the first-line agent and bDMARDs are used as secondary agents, mostly in combination with csDMARDs [10].

This study found that the proportion of patients receiving bDMARD monotherapy in China was only $10.5 \%$, considerably lower than that in other countries. Yazici et al. investigated the RA patient cohort in the Thomson Healthcare MarketScan Research databases and found that $30 \%$ of adults with RA received bDMARD monotherapy in the USA [19]. Gabay et al. examined the data from the Swiss Clinical Quality Management in Rheumatic Diseases registry for RA patients and found that bDMARD monotherapy was prescribed for up to $39 \%$ of treatment courses [21]. Kaufmann
Table 5 The effects of treatment type on DAS28 score and treatment target (low disease activity and remission)

\begin{tabular}{|c|c|c|c|c|c|}
\hline & Patients $^{\mathrm{a}}$ & $\begin{array}{l}\text { Mean DAS28 } \\
\text { score (SD) }\end{array}$ & $p$ value & $\begin{array}{l}\text { Remission + LDA } \\
\text { rate }(\%)\end{array}$ & $p$ value \\
\hline bDMARD therapy & 412 (total) & & & & \\
\hline bDMARD monotherapy & 56 & $4.8(1.8)$ & 0.0108 & 21.4 & 0.507 \\
\hline bDMARD combination therapy & 356 & $4.3(1.5)$ & & 25.6 & \\
\hline bDMARD monotherapy & 56 (total) & & & & \\
\hline TNF inhibitors ${ }^{\mathrm{b}}$ & 49 & $5.0(1.7)$ & 0.0479 & 16.3 & 0.0317 \\
\hline Tocilizumab & 7 & $3.6(2.1)$ & & 57.1 & \\
\hline TNF inhibitors $^{\mathrm{b}}$ & 327 & & & & \\
\hline Monotherapy & 49 & $5.0(1.7)$ & 0.0012 & & \\
\hline Combination therapy & 278 & $4.3(1.4)$ & & & \\
\hline Tocilizumab & 85 & & & & \\
\hline Monotherapy & 7 & $3.6(2.1)$ & 0.2672 & & \\
\hline Combination therapy & 78 & $4.3(1.6)$ & & & \\
\hline
\end{tabular}

${ }^{\text {a }}$ Number of patients with available score data

${ }^{\mathrm{b}}$ TNF inhibitors include infliximab, adalimumab, and etanercept 
et al. conducted a retrospective study of 254 German patients and found that between $18 \%$ and $41 \%$ of patients treated with bDMARDs received the agent as monotherapy [22].

Overall, disease control in our patient cohort was not optimal despite the use of bDMARDs. The majority $(75 \%)$ of patients were classified as having moderate- or high-disease activity per DAS28 scores. The substantially shorter mean treatment duration of bDMARD therapy (4.7-34.5 weeks) in our study population versus that shown in other studies [23, 24] may be responsible for the suboptimal remission rates that we observed. The mean duration of treatment with etanercept, adalimumab, and infliximab in Italian patients with RA from the Italian Group for the Study of Early Arthritis registry was 3.1, 2.6, and 2.7 years, respectively [23]. Patients with RA from the Swiss Clinical Quality Management in Rheumatic Diseases registry received anti-TNF therapy for a median duration of 37 months [24]. The possible reason for the short treatment duration in the Chinese patients in the current study may be associated with poor socioeconomic condition, poor patient compliance, and the limitation of cross-sectional study. bDMARDs are relatively expensive and are not covered by the national health insurance reimbursement policies in China.

This study demonstrated that the proportion of remission + LDA was the highest in patients with $\geq 12$ months of treatment, indicating that long-time treatment maximizes the benefits for patients. These results are in line with those of a previous 4year follow-up study of infliximab therapy in refractory RA patients, which showed that longer duration of infliximab therapy resulted in lower DAS28 score [25]. Nam et al. found that maintenance of clinical responses was higher with bDMARD continuation [26]. Thus, our results suggest that, to achieve the best treatment outcome, patients with RA should continue bDMARD therapy for longer than 12 months. Furthermore, this study revealed that the remission rate in Chinese patients receiving bDMARDs was $12.6 \%$ (DAS28), $5.4 \%$ (CDAI), and 3.5\% (SDAI). In a multicenter cross-sectional study of RA in Chinese patients receiving csDMARDs, bDMARDs, and/or glucocorticoids, Wang et al. found the remission rate to be $8.6 \%$ (DAS28), $8.2 \%$ (CDAI), $8.4 \%$ (SDAI), and $6.8 \%$ (Boolean) [27]. In Qatari, the remission rate of patients with $\mathrm{RA}$ receiving bDMARDs and/or csDMARDs was $49 \%$ by DAS28 score [28]. In the QUEST-RA study including patients with RA from 25 mostly European countries, remission rate was $13.8 \%$ and $19.6 \%$ by CDAI and DAS28, respectively [29]. These results indicate that treatment for Chinese patients with RA is insufficient. Further, the inhibitory costs of biologics $(\$ 15,000-\$ 25,000$ per patient per year) [30] present challenges in developing countries [31] and may result in early treatment discontinuation or dose reduction, resulting in flaring of the disease. While the guidelines do not recommend treatment discontinuation, "step-down" or tapering strategies by careful dose reduction or injection spacing can maintain a disease-free status in patients who have achieved remission or low disease activity [32]. Studies have shown that patients in remission after bDMARD mono or combination therapy continued to maintain their remission status after careful dose tapering or treatment discontinuation [33, 34]. Further, in order to maintain the alleviating effect, the dose of traditional DMARDS may be increased or treatment options such as double and triple combination treatment may be prescribed. Therapy can be reinitiated when necessary, making this approach desirable from a safety viewpoint and curbing unnecessary healthcare expenditure. Careful patient selection based on clinical and practical considerations may also improve outcomes with continuous bDMARD use in the developing world.

The current RA management regimen in China should be optimized to allow patients to benefit from more aggressive treatment. Given the comparable efficacy and costeffectiveness of triple DMARD combinations in comparison with bDMARDs and methotrexate $[35,36]$, further studies are needed to assess the use of triple DMARD combination therapy in comparison with biologics in China.

Although the 2013 EULAR guidelines for RA management recommend bDMARDs to be used concomitantly with csDMARDs [10], the 2015 ACR guidelines take monotherapy into consideration [37] and bDMARDs monotherapy is also used in clinical practice as needed [12]. In some studies, bDMARD monotherapy has been shown to be as effective as the combination therapy of csDMARDs and bDMARDs. In terms of DAS28 score, ACR responses, and swollen and tender joint counts, tocilizumab and methotrexate combination therapy was not superior to tocilizumab monotherapy [13, 38]. Consistently, the current study also demonstrated that DAS28 score in patients receiving tocilizumab monotherapy was similar to that in patients receiving tocilizumab and csDMARD combination therapy. In the current study, although the average DAS28 score was significantly higher in patients receiving bDMARD monotherapy than in patients receiving combination therapy, the rate of reaching treatment target was similar in patients receiving monotherapy versus combination therapy. Interestingly, the current study found that patients with tocilizumab monotherapy showed significantly lower DAS28 score and higher proportion of reaching treatment target than patients receiving other types of bDMARD monotherapy. These findings suggest that tocilizumab monotherapy may be a promising option for patients with RA. Simplification and optimization of the treatment for RA are beneficial for both patients and physicians.

This cross-sectional study design presents a "snapshot" of bDMARD usage in Chinese patients with RA. It is difficult to infer causal relationships between bDMARD usage pattern and disease activity of RA, although longer duration of bDMARD therapy was associated with lower DAS28 score and higher rate of achievement of treatment goal through ad hoc analysis. Confounding factors that might affect the 
relationship between duration of bDMARD therapy and disease activity, such as the disease status prior to receiving bDMARD therapy and other concomitant therapies, were not considered in this study.

In conclusion, the usage of bDMARDs in Chinese patients with RA is in accordance with the global recommendations despite the short treatment duration. The bDMARDs are commonly used in combination with csDMARDs. Longer duration of bDMARD therapy appears to be associated with lower DAS28 scores and higher proportion of achievement of treatment target. These results should be kept in mind by the clinicians while treating patients with RA who do not achieve the treatment goal within 3 to 6 months of therapy. To achieve the best treatment outcome, patients with RA should continue bDMARD therapy for longer than 12 months. Our results provide a strong evidence base for the government to make decisions for the benefit of a greater number of patients with RA. Tocilizumab monotherapy may be a promising option for patients with RA. Further prospective studies are needed to assess the impact of bDMARD usage on RA remission among the Chinese population.

Acknowledgments This study was sponsored by Shanghai Roche Pharmaceuticals Ltd. Medical writing services were provided by Cactus Communications and paid for by Shanghai Roche Pharmaceuticals Ltd., China. The authors retained full control of manuscript content.

Compliance with ethical standards The study was approved by the Institutional Review Board of Peking University and independent ethics committees responsible for each investigating site. The study was conducted in compliance with the Declaration of Helsinki and in accordance with Good Clinical Practice and relevant ethical guidelines. Written informed consent was obtained from each participant.

Disclosures Jing Liu and Yu Bi are employees of Shanghai Roche Pharmaceutical Ltd., and funding for this study was provided by Shanghai Roche Pharmaceutical Ltd.

Open Access This article is distributed under the terms of the Creative Commons Attribution 4.0 International License (http:// creativecommons.org/licenses/by/4.0/), which permits unrestricted use, distribution, and reproduction in any medium, provided you give appropriate credit to the original author(s) and the source, provide a link to the Creative Commons license, and indicate if changes were made.

\section{References}

1. Cross M, Smith E, Hoy D et al (2014) The global burden of rheumatoid arthritis: estimates from the global burden of disease 2010 study. Ann Rheum Dis 73:1316-1322. doi:10.1136/annrheumdis2013-204627

2. Woolf AD, Pfleger B (2003) Burden of major musculoskeletal conditions. Bull World Health Organ 81:646-656

3. Li R, Sun J, Ren LM et al (2012) Epidemiology of eight common rheumatic diseases in China: a large-scale cross-sectional survey in Beijing. Rheumatology (Oxford) 51:721-729. doi:10.1093 /rheumatology/ker370
4. Zeng QY, Chen R, Darmawan J et al (2008) Rheumatic diseases in China. Arthritis Res Ther 10:R17. doi:10.1186/ar2368

5. Zhang X, Mu R, Wang X et al (2015) The impact of rheumatoid arthritis on work capacity in Chinese patients: a cross-sectional study. Rheumatology (Oxford) 54:1478-1487. doi:10.1093 /rheumatology/kev014

6. Li ZG (2015) A new look at rheumatology in China-opportunities and challenges. Nat Rev Rheumatol 11:313-317. doi:10.1038 /nrrheum.2014.218

7. Xu C, Wang X, Mu R et al (2014) Societal costs of rheumatoid arthritis in China: a hospital-based cross-sectional study. Arthritis Care Res (Hoboken) 66:523-531. doi:10.1002/acr.22160

8. Chou CT, Pei L, Chang DM, Lee CF, Schumacher HR, Liang MH (1994) Prevalence of rheumatic diseases in Taiwan: a population study of urban, suburban, rural differences. J Rheumatol 21:302306. doi:10.1002/acr.21820

9. Jiang L, Yin J, Ye L, et al. (2014) Novel risk loci for rheumatoid arthritis in Han Chinese and congruence with risk variants in Europeans. Arthritis Rheumatol 66:1121-32. Erratum in: Arthritis Rheumatol (2014) 66:1881. doi:10.1002/art.38353

10. Smolen JS, Landewé R, Breedveld FC et al (2014) EULAR recommendations for the management of rheumatoid arthritis with synthetic and biological disease-modifying antirheumatic drugs: 2013 update. Ann Rheum Dis 73:492-509. doi:10.1136/annrheumdis2013-204573

11. Singh JA, Furst DE, Bharat A et al (2012) 2012 Update of the 2008 American College of Rheumatology recommendations for the use of disease-modifying antirheumatic drugs and biologic agents in the treatment of rheumatoid arthritis. Arthritis Care Res (Hoboken) 64: 625-639. doi:10.1002/acr.21641

12. Emery P, Sebba A, Huizinga TW (2013) Biologic and oral diseasemodifying antirheumatic drug monotherapy in rheumatoid arthritis. Ann Rheum Dis 72:1897-1904. doi:10.1136/annrheumdis-2013203485

13. Dougados M, Kissel K, Sheeran T et al (2013) Adding tocilizumab or switching to tocilizumab monotherapy in methotrexate inadequate responders: 24-week symptomatic and structural results of a 2 year randomized controlled strategy trial in rheumatoid arthritis (ACT-RAY). Ann Rheum Dis 272:43-50. doi:10.1136 /annrheumdis-2011-201282

14. Gabay C, Riek M, Hetland ML et al (2015) Effectiveness of tocilizumab with and without synthetic disease-modifying antirheumatic drugs in rheumatoid arthritis: results from a European collaborative study. Ann Rheum Dis. doi:10.1136/annrheumdis-2015207760

15. Arnett FC, Edworthy SM, Bloch DA et al (1988) The American Rheumatism Association 1987 revised criteria for the classification of rheumatoid arthritis. Arthritis Rheum 31:315-324. doi:10.1002 /art.1780310302

16. Prevoo ML, van't Hof MA, Kuper HH, van Leeuwen MA, van de Putte LB, van Riel PL (1995) Modified activity scores that include twenty-eight-joint counts. Development and validation in a prospective longitudinal study of patients with rheumatoid arthritis. Arthritis Rheum 38:44-48. doi:10.1002/art.1780380107

17. Mäkinen H, Kautiainen H, Hannonen P, Sokka T (2005) Is DAS28 an appropriate tool to assess remission in rheumatoid arthritis? Ann Rheum Dis 64:1410-1413. doi:10.1136/ard.2005.037333

18. Soliman MM, Ashcroft DM, Watson KD, Lunt M, Symmons DP, Hyrich KL (2011) British Society for Rheumatology Biologics Register. Impact of concomitant use of DMARDs on the persistence with anti-TNF therapies in patients with rheumatoid arthritis: results from the British Society for Rheumatology Biologics Register. Ann Rheum Dis 70:583589. doi:10.1136/ard.2010.139774

19. Yazici Y, Shi N, John A (2008) Utilization of biologic agents in rheumatoid arthritis in the United States: analysis of prescribing 
patterns in 16,752 newly diagnosed patients and patients new to biologic therapy. Bull NYU Hosp Jt Dis 66:77-85

20. Jia Y, Chen X, Wang Y, Li W, Li Y (2007) Standardization of values of laboratory tests from different clinical centers. Chin J Dis Control Prev 11:435-436

21. Gabay C, Hasler P, Kyburz D et al (2014) Biological agents in monotherapy for the treatment of rheumatoid arthritis. Swiss Med Wkly 144:w13950. doi:10.4414/smw.2014.13950

22. Kaufmann J, Feist E, Roske AE, Schmidt WA (2013) Monotherapy with tocilizumab or TNF-alpha inhibitors in patients with rheumatoid arthritis: efficacy, treatment satisfaction, and persistence in routine clinical practice. Clin Rheumatol 32:1347-1355. doi:10.1007 /s10067-013-2281-8

23. Iannone F, Gremese E, Atzeni F et al (2012) Longterm retention of tumor necrosis factor- $\alpha$ inhibitor therapy in a large Italian cohort of patients with rheumatoid arthritis from the GISEA registry: an appraisal of predictors. J Rheumatol 39:1179-1184. doi:10.3899/jrheum.111125

24. Du Pan SM, Dehler S, Ciurea A, Ziswiler HR, Gabay C, Finckh A (2009) Swiss Clinical Quality Management Physicians. Comparison of drug retention rates and causes of drug discontinuation between anti-tumor necrosis factor agents in rheumatoid arthritis. Arthritis Rheum 61:560-568. doi:10.1002/art.24463

25. Vander Cruyssen B, Van Looy S, Wyns B et al (2006) Four-year follow-up of infliximab therapy in rheumatoid arthritis patients with long-standing refractory disease: attrition and long-term evolution of disease activity. Arthritis Res Ther 8:R112

26. Nam JL, Ramiro S, Gaujoux-Viala C et al (2014) Efficacy of biological disease-modifying antirheumatic drugs: a systematic literature review informing the 2013 update of the EULAR recommendations for the management of rheumatoid arthritis. Ann Rheum Dis 73:516-528. doi:10.1136/annrheumdis-2013-204577

27. Wang GY, Zhang SL, Wang XR et al (2015) Remission of rheumatoid arthritis and potential determinants: a national multi-center cross-sectional survey. Clin Rheumatol 34:221-230. doi:10.1007 /s10067-014-2828-3

28. Lutf A, Poil AR, Hammoudeh M (2014) Characteristics of patients with rheumatoid arthritis in Qatar: a cross-sectional study. Int $\mathrm{J}$ Rheum Dis 17:63-65. doi:10.1111/1756-185X.12135

29. Sokka T, Kautiainen H, Pincus T et al (2009) Disparities in rheumatoid arthritis disease activity according to gross domestic product in 25 countries in the QUEST-RA database. Ann Rheum Dis 68: 1666-1672. doi:10.1136/ard.2009.109983

30. Roberts L, McColl GJ (2004) Tumour necrosis factor inhibitors: risks and benefits in patients with rheumatoid arthritis. Intern Med J 34:687-693

31. Darmawan J, World Health Organization-International League of Associations for Rheumatology Community Oriented Program for Control of Rheumatic Disease (2007) Recommendations from the Community Oriented Program for Control of Rheumatic Disease for data collection for the measurement and monitoring of health in developing countries. Clin Rheumatol 26:853-857

32. Fautrel B, Den Broeder AA (2015) De-intensifying treatment in established rheumatoid arthritis (RA): why, how, when and in whom can DMARDs be tapered? Best Pract Res Clin Rheumatol 29:550-565. doi:10.1016/j.berh.2015.09.006

33. Haschka J, Englbrecht M, Hueber AJ et al (2016) Relapse rates in patients with rheumatoid arthritis in stable remission tapering or stopping antirheumatic therapy: interim results from the prospective randomised controlled RETRO study. Ann Rheum Dis 75:45-51. doi:10.1136/annrheumdis-2014-206439

34. Emery P, Hammoudeh M, FitzGerald O et al (2014) Sustained remission with etanercept tapering in early rheumatoid arthritis. N Engl J Med 371:1781-1792. doi:10.1056/NEJMoa1316133

35. Moreland LW, O'Dell JR, Paulus HE et al (2012) A randomized comparative effectiveness study of oral triple therapy versus etanercept plus methotrexate in early aggressive rheumatoid arthritis: the treatment of Early Aggressive Rheumatoid Arthritis Trial. Arthritis Rheum 64:2824-2835. doi:10.1002/art.34498

36. Sethi MK, O'Dell JR (2015) Combination conventional DMARDs compared to biologicals: what is the evidence? Curr Opin Rheumatol 27:183-188. doi:10.1097/BOR.0000000000000153

37. Singh JA, Saag KG, Bridges SL Jr et al (2016) 2015 American college of rheumatology guideline for the treatment of rheumatoid arthritis. Arthritis Rheum 68:1-26. doi:10.1002/art.39480

38. Dougados M, Kissel K, Conaghan PG et al (2014) Clinical, radiographic, and immunogenic effects after 1 year of tocilizumab based treatment strategy with and without methotrexate in rheumatoid arthritis: the ACT RAY Study. Ann Rheum Dis 73:803-809. doi:10.1136/annrheumdis-2013-204761 